\title{
Pregnancy outcomes of normal versus abnormal cardiotocography in a tertiary centre in Nepal
}

\author{
Mariyam Faruqi*, Shailaja C. Shrestha, Rabindra D. Bhatta
}

Department of Obstetrics and Gynecology, B. P. Koirala Institute of Health Sciences, Dharan, Nepal

Received: 03 September 2019

Accepted: 04 October 2019

*Correspondence:

Dr. Mariyam Faruqi,

E-mail: faruqimariyam@gmail.com

Copyright: (c) the author(s), publisher and licensee Medip Academy. This is an open-access article distributed under the terms of the Creative Commons Attribution Non-Commercial License, which permits unrestricted non-commercial use, distribution, and reproduction in any medium, provided the original work is properly cited.

\begin{abstract}
Background: Pregnancy and childbirth is normal physiological process with great pathological potential. The obstetricians are more concerned with the early recognition of fetal distress during labour and such an adverse outcome should be detected at the earliest point of time by an effective surveillance method. Cardiotocography as a part of biophysical profile has become an established diagnostic tool for fetal surveillance. To compare early perinatal outcome of normal and abnormal cardiotocography in terms of APGAR scores, need for neonatal resuscitation, NICU admission, perinatal death and mode of delivery.

Methods: It was a cross-sectional study. 200 nulliparous/multiparous women with singleton pregnancy in cephalic presentation at gestational age 37-42 weeks in latent stage of labor were enrolled in the study and subjected to admission test in left lateral position using fetal monitor. Baseline FHR and contraction pattern were determined for 20 minutes and classification of patients was done into normal, and suspicious or abnormal according to the FIGO guidelines 2015.

Results: Low APGAR scores, rate of LSCS, need for neonatal resuscitation, neonatal admission were more in the abnormal cardiotocography group. Cardiotocography in the current study has high sensitivity and high negative predictive value for detecting fetal distress.

Conclusions: From the analysis of this study, it would be safe to conclude that an ominous cardiotocography should be managed appropriately without delay and obstetrician should be vigilant in suspicious as well as in normal admission test group for timely intervention for bettering the neonatal outcome.
\end{abstract}

Keywords: APGAR, Cardiotocography, Intrapartum fetal surveillance, Loop of cord around fetal neck Pregnancy outcome, Meconium stained liquor, Neonatal resuscitation, NICU admission

\section{INTRODUCTION}

Pregnancy and childbirth are normal physiological process with great pathological potential. Most of the pregnancies progress normally but some pregnancies are more complex. The antenatal and intra-partum conditions place the mother, or the developing fetus or both at a higher risk for complications. ${ }^{1}$ Some instances can lead to adverse outcome resulting in immediate and late neonatal and maternal problems.
The obstetricians are more concerned with the early recognition of fetal distress during labour as it's a common occurrence and such an adverse outcome should be detected at the earliest point of time by an effective surveillance method.

Intermittent auscultation, continuous electronic fetal heart rate (FHR) monitoring and invasive techniques like fetal blood gas analysis are the available methods for such surveillance. ${ }^{2}$ 
Non stress test as a part of biophysical profile of antepartum fetal heart rate monitoring has become an established diagnostic tool for fetal surveillance in high risk pregnancy. The ease of administration, lack of contradiction, less expense, easy interpretability makes cardiotocography in labour the primary means of surveillance. The concept of CTG in labour using fetal heart rate monitoring has been popularized to serve as a form of intrapartum risk assessment scheme using the basic principle of correlating the accelerations of fetal heart rate with fetal movement as an indicator of intact responsive central nervous system. ${ }^{3}$

Cardiotocography is most diffuse, non-invasive pre-natal diagnostic technique to monitor fetal health during labour. It records the fetal heart rate in relation to maternal uterine contractions for about 20 to 30 minutes and is one of the most common forms of fetal assessment during labour. ${ }^{4}$ The rationale for electronic fetal monitoring (EFM) is based on the knowledge that when normal metabolic processes are interrupted, either by lack of oxygen $(\mathrm{O} 2)$ or an inability to expel end products, the subsequent accumulation of acids may damage all or part of the living system. ${ }^{5}$

The studies related to the use of cardiotocography as a screening tool to identify subgroup of fetuses that would benefit from more intensive monitoring, early identification of clinically inapparent fetal hypoxia leading to an early intervention thereby preventing the adverse fetal outcome are scarce in Nepal. So, this study was undertaken with the aim to correlate abnormal cardiotocography and its perinatal outcome.

\section{METHODS}

This study was conducted at B. P. Koirala Institute of Health Sciences, Dharan, Nepal to compare normal and abnormal cardiotocography with early neonatal outcomes, perinatal death, APGAR scores, need for neonatal resuscitation and NICU admission. A cross sectional study conducted over 12 months (March 2016 to March 2017).

Pregnant women who were admitted to antenatal ward with period of gestation between $37-42$ week as per the last menstrual period or the 1 st trimester obstetric scan meeting the inclusion criteria were enrolled in the study.

\section{Inclusion criteria}

- Nulliparous/multiparous women

- Singleton pregnancy

- Cephalic presentation

- Gestational age 37-42 weeks as determined from the date of the last menstrual period and/or confirmed by first trimester ultrasound measurements

- Those patients who came in latent stage of labor.

\section{Exclusion criteria}

- Those who refuse to participate in the study

- Malpresentation

- Intrauterine growth restriction

- Gestational diabetes

- Obstetric cholestasis

- Previous uterine surgery

- Severe oligohydramnios (AFI less than 5)

- Abruptio placenta

- Preeclampsia

- Multiple pregnancy

- Known gross congenital malformation in the fetus.

Sample size was calculated from a similar study by Shrutiprabha et al where abnormal and normal CTG was compared with fetal outcomes. ${ }^{6}$

- According to the data collected.

Using formula of double proportion sample size was calculated as:

$$
n=\frac{2(\bar{p})(1-\bar{p})\left(Z_{\beta}+Z_{\alpha / 2}\right)^{2}}{\left(\mathrm{p}_{1}-p_{2}\right)^{2}}
$$

$\mathrm{n}=86.6$

- Rounding off to the nearest whole number $=87$ in each group

- Taking dropouts+ lost cases into account sample size $=100$ in each group

- $\quad$ Final sample size $=100$ in each group

Written informed consent was taken to participate in the study. Information was collected from subjects via a questionnaire and demographic data was collected by the investigator including the duration of the condition, antenatal events, significant past or family history, previous pregnancy outcomes and detailed examination of the patient was done.

Patients were subjected to admission test using fetal monitor for 20 minutes after ensuring maternal hydration and food intake.

The patient was put in left lateral position to minimize supine hypotension.

The transducer was placed on the maternal abdomen at a site where fetal heart action was best detected. A coupling gel was applied because air conducts ultrasound poorly. The device was held in position by a belt. Indirect assessment of uterine contraction was possible through an external tocographic transducer. Baseline FHR and contraction pattern were determined for 20 minutes in all the patients who came in latent stage of labor. These patients were followed up to delivery by intermittent 
auscultation. Important events during labor like development of fetal distress on auscultation and passage of meconium were noted.

Classification of patients with the cardiotocography results was done into normal, and suspicious or abnormal according to the FIGO guidelines 2015.

\section{Criteria for normal cardiotocography}

- At least 2 acceleration (>15 bpm for $>15 \mathrm{sec})$

- Baseline heart rate: $110-160 \mathrm{bpm}$

- Baseline variability: 5-25 bpm

- Absence of decelerations

- Moderate tachycardia (161-180 bpm)/ bradycardia (100-109 bpm) but with preservation of baseline variability and accelerations.

\section{Criteria for suspicious cardiotocography}

Lacking at least one characteristic of normality, but with no pathological features.

\section{Criteria for abnormal cardiotocography}

- Silent baseline variability $(<5 \mathrm{bpm})$ pattern $>40$ minutes

- Baseline heart rate $>180 \mathrm{bpm}$ or $<100 \mathrm{bpm}$

- Late decelerations

- Atypical/ significant variable decelerations (depth $>60 \mathrm{bpm}$ and duration $>60 \mathrm{sec}$ )

- Prolonged bradycardia (drop of the fetal heart rate $<100 \mathrm{bpm}$ for $>3$ minutes or $<80 \mathrm{bpm}$ for $>2$ minutes).

\section{Sinusoidal pattern >10 minutes}

All the patients with abnormal CTG as per the criteria mentioned above were enrolled as cases.

In case of suspicious pattern, the CTG was repeated as per the standard practice followed in this institution (i.e. after $500 \mathrm{ml}$ of IV fluid and oxygen). If it still remained suspicious it was taken as abnormal CTG. Then these patients were also enrolled as cases.

Age and Gravidity and parity matched women with normal CTG in latent stage of labor were taken as control in 1:1 basis. Fetal and maternal outcomes were noted.

\section{Statistical analysis}

After completion of study data was statistically described in frequencies (number of cases) and percentages (\%) when appropriate.

The relevant data were entered in Microsoft Excel software 2013 from the proforma. All the data were exported to the software SPSS version 11.5 and analysis was done. Chi square test was used for comparison of data for statistical significance.

For descriptive statistics percentage, mean and standard deviation was calculated.

\section{RESULTS}

Two hundred pregnant women in labor admitted to obstetrics ward of B.P. Koirala Institute of Health Sciences, Dharan, Nepal were enrolled for the present study.

The number of patients with normal cardiotocography was 100 and abnormal cardiotocography was 100 .

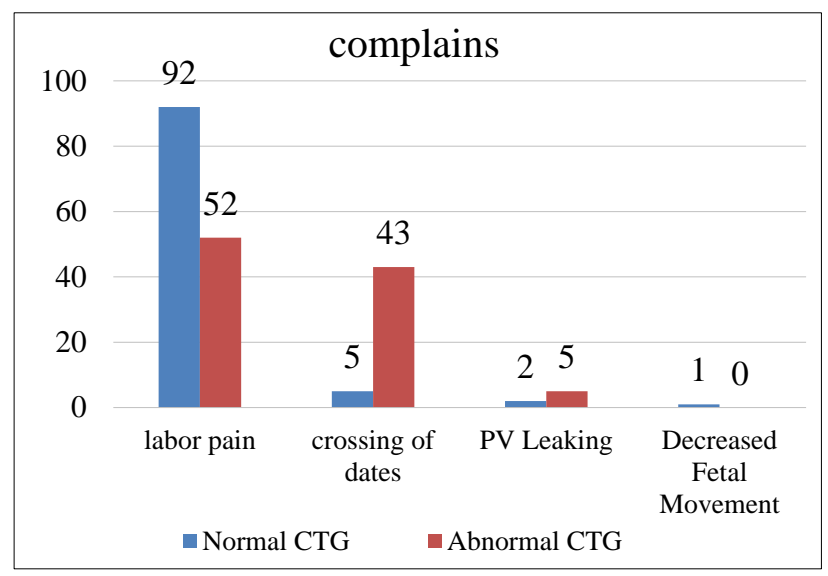

Figure 1: Complains of the patients at the time of admission in the two study groups.

Table 1: Baseline characteristics in the two study groups.

\begin{tabular}{|lll|}
\hline Baseline data & $\begin{array}{l}\text { Mean } \pm \text { SD } \\
\text { Normal } \\
\text { CTG }\end{array}$ & $\begin{array}{l}\text { Abnormal } \\
\text { CTG }\end{array}$ \\
\hline Booked & $40 \pm 0$ & $47 \pm 0$ \\
\hline Unbooked & $51 \pm 0$ & $45 \pm 0$ \\
\hline Registered & $9 \pm 0$ & $8 \pm 0$ \\
\hline Maternal age (years) & $24.41 \pm 4.20$ & $24.93 \pm 4.60$ \\
\hline Parity & $1.6 \pm 0.670$ & $1.59 \pm 0.880$ \\
\hline $\begin{array}{l}\text { Gestational age } \\
\text { (weeks) }\end{array}$ & $39.52 \pm 1.190$ & $39.99 \pm 1.280$ \\
\hline $\begin{array}{l}\text { Cervical dilatation } \\
\text { (cm) }\end{array}$ & $1.76 \pm 0.520$ & $1.615 \pm 0.840$ \\
\hline Bishop Score & $3.53 \pm 0.834$ & $3.52 \pm 0.785$ \\
\hline Cord Length $(\mathrm{cm})$ & $46.73 \pm 7.80$ & $46.8 \pm 8.50$ \\
\hline Birth weight $(\mathrm{kg})$ & $3.08 \pm 0.465$ & $2.98 \pm 0.428$ \\
\hline
\end{tabular}

In the normal cardiotocography group 40 cases were booked, 51 cases were unbooked and 9 were registered cases. While in the abnormal CTG group there were 47, 45 and 8 cases in the respective groups. The baseline data which includes booking status, maternal age, parity, gestational age, cervical dilatation, bishop score, cord 
length and birthweight of baby is comparable in the two study groups Table 1 .

Majority of the patients in both the groups had complains of labor pain at the time of admission followed by crossing of dates, per vaginum leaking and decreased fetal movement Figure 1.
APGAR scores of the babies in the normal CTG group at 1 minute was more than 7 in $98 \%$ cases, between 5 to 6 in $2 \%$ cases and less than 5 in $0 \%$ cases as compared to that in the abnormal group, which was $92 \%, 5 \%$ and $3 \%$ respectively. This was statistically significant with a $\mathrm{p}$ value of 0.047 .

Table 2: APGAR score of the new-born at 1, 5 and 10 minutes.

\begin{tabular}{|c|c|c|c|c|c|c|}
\hline \multicolumn{7}{|c|}{ APGAR Score } \\
\hline & & $\begin{array}{l}\text { Normal CTG (Control) } \\
n=100(50.0 \%)\end{array}$ & $\begin{array}{l}\text { Abnormal CTG } \\
(\text { case }) n=100(50.0 \%)\end{array}$ & Total $n=200$ & $\begin{array}{l}X^{2} \\
\text { value }\end{array}$ & p-value \\
\hline \multirow{3}{*}{ At 1 min } & $<5$ & $0(0.0 \%)$ & $3(3.0 \%)$ & $3(1.50 \%)$ & \multirow{3}{*}{4.475} & \multirow{3}{*}{0.047} \\
\hline & $5-6$ & $2(2.0 \%)$ & $5(5.0 \%)$ & $7(3.50 \%)$ & & \\
\hline & $>7$ & $98(98.0 \%)$ & $92(92.0 \%)$ & $190(95.0 \%)$ & & \\
\hline \multirow{3}{*}{ At $5 \mathrm{~min}$} & $<5$ & $0(0.0 \%)$ & $1(0.50 \%)$ & $1(0.50 \%)$ & \multirow{3}{*}{4.082} & \multirow{3}{*}{0.041} \\
\hline & $5-6$ & $0(0.0 \%)$ & $3(3.0 \%)$ & $3(1.50 \%)$ & & \\
\hline & $>7$ & $100(100 \%)$ & $96(96.0 \%)$ & $196(98.0 \%)$ & & \\
\hline \multirow{3}{*}{ At 10 min } & $<5$ & $0(0.0 \%)$ & $1(0.50 \%)$ & $1(0.50 \%)$ & \multirow{3}{*}{3.046} & \multirow{3}{*}{0.079} \\
\hline & $5-6$ & $0(0.0 \%)$ & $2(2.0 \%)$ & $2(1.0 \%)$ & & \\
\hline & $>7$ & $100(100 \%)$ & $97(97.0 \%)$ & $197(98.50 \%)$ & & \\
\hline
\end{tabular}

Table 3: Need of resuscitation in the two study groups.

\begin{tabular}{|c|c|c|c|c|c|}
\hline \multicolumn{6}{|c|}{ Need for neonatal resuscitation } \\
\hline & $\begin{array}{l}\text { Normal CTG (control) } \\
\mathbf{n}=100(\mathbf{5 0 . 0} \%)\end{array}$ & $\begin{array}{l}\text { Abnormal CTG (case) } \\
\mathrm{n}=100(50.0 \%)\end{array}$ & Total $\mathbf{n}=\mathbf{2 0 0}$ & $X^{2}$ value & p-value \\
\hline Resuscitated & $2(2.0 \%)$ & $12(12.0 \%)$ & $14(7.0 \%)$ & & \\
\hline Not resuscitated & $98(98.0 \%)$ & $88(88.0 \%)$ & $186(93.0 \%)$ & 7.680 & 0.005 \\
\hline
\end{tabular}

Table 4: Neonatal admission in the two study groups.

\begin{tabular}{|c|c|c|c|c|c|}
\hline \multicolumn{6}{|c|}{ Neonatal admission } \\
\hline & $\begin{array}{l}\text { Normal CTG } \\
(\text { control) } n=100(50.0 \%)\end{array}$ & $\begin{array}{l}\text { Abnormal CTG (case) } \\
n=100(50.0 \%)\end{array}$ & Total $n=200$ & $\mathrm{X} 2$ value & p-value \\
\hline Admitted & $1(1.0 \%)$ & $12(12.0 \%)$ & $13(6.50 \%)$ & \multirow{2}{*}{9.955} & \multirow{2}{*}{0.001} \\
\hline Not admitted & $99(99.0 \%)$ & $88(88.0 \%)$ & $187(93.50 \%)$ & & \\
\hline
\end{tabular}

Table 5: Fetal outcome in the study group.

\begin{tabular}{|c|c|c|c|c|c|}
\hline \multicolumn{6}{|c|}{ Fetal outcome } \\
\hline & $\begin{array}{l}\text { Normal CTG (control) } \\
\mathbf{n}=100(50.0 \%)\end{array}$ & $\begin{array}{l}\text { Abnormal CTG (case) } \\
n=100(50.0 \%)\end{array}$ & Total $\mathbf{n}=\mathbf{2 0 0}$ & $\mathrm{X}^{2}$ value & p-value \\
\hline Alive & $100(100 \%)$ & $99(99.0 \%)$ & $199(99.50 \%)$ & \multirow{2}{*}{1.005} & \multirow{2}{*}{0.315} \\
\hline Still Birth & $0(0.0 \%)$ & $1(1.0 \%)$ & $1(0.50 \%)$ & & \\
\hline
\end{tabular}

APGAR scores of the babies in the normal CTG group at 5 minutes was more than 7 in $100 \%$ cases, between 5 to 6 in $0 \%$ cases and less than 5 in $0 \%$ cases compared to that in the abnormal group, which was $96 \%, 3 \%$ and $1 \%$ respectively, with a $\mathrm{p}$ value of 0.041 .

The APGAR scores of $100(100 \%)$ babies in the normal CTG group at 10 minutes was more than 7 whereas $97 \%$ babies had APGAR scores greater than 7,2\% between 5 to 6 and only $1 \%$ less than 5 in the abnormal group, with a p value of 0.079 Table 2 .

$12(12 \%)$ babies among abnormal group and $2(2 \%)$ among normal group needed resuscitation in the form of bag and mask ventilation with a statistically significant $p$ value of 0.005 Table 3 . 
$12(12 \%)$ babies among abnormal group and 1(1\%) among normal group got admitted in neonatal ICU. This was statistically significant with a $\mathrm{p}$ value of 0.001 Table 4. Only $1(1.0 \%)$ patient of the abnormal cardiotocography group had a stillbirth, while the remaining $99(99.0 \%)$ patients had live births. None of the patients in the normal group had stillbirths, all had live born babies Table 5 .
In the abnormal CTG group 90 (90\%) cases had caesarean section, $9(9 \%)$ had normal vaginal delivery and only $1(1 \%)$ had instrumental delivery whereas $90(90 \%)$ had normal vaginal delivery, 7 (7\%) had caesarean section and only $3(3 \%)$ had instrumental delivery in normal CTG group. The $\mathrm{p}$ value being 0.0 which is statistically significant Table 6.

Table 6: Mode of delivery in the study groups.

\begin{tabular}{|c|c|c|c|c|c|}
\hline \multicolumn{6}{|c|}{ Mode of delivery } \\
\hline & $\begin{array}{l}\text { Normal CTG (control) } \\
n=100(50.0 \%)\end{array}$ & $\begin{array}{l}\text { Abnormal CTG } \\
\text { (case) } n=100(50.0 \%)\end{array}$ & Total $n=200$ & $X^{2}$ value & p-value \\
\hline SVD & $90(90.0 \%)$ & $9(9.0 \%)$ & $99(49.50 \%)$ & \multirow{3}{*}{138.3} & \multirow{3}{*}{0.000} \\
\hline Instrumental delivery & $3(3.0 \%)$ & $1(1.0 \%)$ & $4(2.0 \%)$ & & \\
\hline LSCS & $7(7.0 \%)$ & $90(90.0 \%)$ & $97(48.50 \%)$ & & \\
\hline
\end{tabular}

Table 7: Meconium stained liquor in the study groups.

\begin{tabular}{|c|c|c|c|c|c|}
\hline \multicolumn{6}{|c|}{ Meconium stained liquor } \\
\hline & $\begin{array}{l}\text { Normal CTG (control) } \\
\mathbf{n}=\mathbf{1 0 0}(\mathbf{5 0 . 0 \%})\end{array}$ & $\begin{array}{l}\text { Abnormal CTG (case) } \\
\mathrm{n}=\mathbf{1 0 0}(\mathbf{5 0 . 0 \%})\end{array}$ & Total $n=200$ & $\begin{array}{l}X^{2} \\
\text { value }\end{array}$ & p-value \\
\hline Meconium stained liquor & $20(20 \%)$ & $60(60.0 \%)$ & $80(40.0 \%)$ & \multirow{2}{*}{33,33} & \multirow{2}{*}{0.0001} \\
\hline No meconium stained liquor & $80(80.0 \%)$ & $40(40.0 \%)$ & $120(60.0 \%)$ & & \\
\hline
\end{tabular}

Table 8: Presence of cord around fetal neck.

\begin{tabular}{|c|c|c|c|c|c|}
\hline \multicolumn{6}{|c|}{ Cord around neck } \\
\hline & $\begin{array}{l}\text { Normal CTG (control) } \\
\mathbf{n}=100(50.0 \%)\end{array}$ & $\begin{array}{l}\text { Abnormal CTG (case) } \\
\mathrm{n}=100(50.0 \%)\end{array}$ & Total $n=200$ & $\mathrm{X}^{2}$ value & p-value \\
\hline Cord around neck & $17(17 \%)$ & $90(90.0 \%)$ & $80(40.0 \%)$ & & \\
\hline No cord around neck & $10(10.0 \%)$ & $83(83.0 \%)$ & $120(60.0 \%)$ & 2.098 & $0.14 / 5$ \\
\hline
\end{tabular}

Table 9: Diagnostic utility of cardiotocography as admission test in labor.

\begin{tabular}{|lllll|}
\hline Cardiotocography & Sensitivity & Specificity & Positive predictive value & Negative predictive value \\
\hline Need for resuscitation & $85.7 \%$ & $52.7 \%$ & $12.0 \%$ & $2.0 \%$ \\
\hline Need of admission & $92.3 \%$ & $52.9 \%$ & $12.0 \%$ & $99.0 \%$ \\
\hline SVD versus operative delivery & $90.0 \%$ & $91.0 \%$ & $90.90 \%$ & $90.10 \%$ \\
\hline Meconium stained liquor & $75.0 \%$ & $66.6 \%$ & $60.0 \%$ & $80.0 \%$ \\
\hline Cord around neck & $63.0 \%$ & $52.0 \%$ & $17.0 \%$ & $90.0 \%$ \\
\hline
\end{tabular}

The $80(40 \%)$ patients had meconium stained liquor of which $60(75 \%)$ belonged to the abnormal CTG group and 20 (25\% belonged to the normal CTG group. This was statistically significant with a $\mathrm{p}$ value of 0.0001 Table 7. Among the 200 patients $17(17 \%)$ women in the abnormal CTG group and $10(10 \%)$ women in the normal CTG group had single or multiple loops of umbilical cord wound around fetal neck. This was not statistically significant. ( $\mathrm{p}$ value 0.14 ) Table 8 .

Taking the group of abnormal cardiotocography as cases and normal cardiotocography as control the sensitivity, specificity, positive predictive value and negative predictive value of abnormal cardiotocography to detect need for resuscitation, need of admission, mode of delivery, presence of meconium stained liquor, cord around the neck were calculated and the results are tabulated Table 9.

\section{DISCUSSION}

Majority of the patients were between 20-30 years of age in both the groups. $40.0 \%$ patients were booked cases, $51 \%$ were unbooked and $9.0 \%$ were registered cases in 
the normal group which was comparable with that in abnormal group, $47 \%, 45 \%$ and $8 \%$ respectively.

Most patients were primigravida and primipara in term pregnancy in latent labor in both the groups.

APGAR scores of the babies in the normal CTG group at 1 minute was more than 7 in $98 \%$ cases, between 5 to 6 in $2 \%$ cases and less than 5 in $0 \%$ cases while the same was $92 \%, 5 \%$ and $3 \%$ respectively in the abnormal group in our study. Results of studies done by Behuria, Goyal and Khursheed were comparable with our results. ${ }^{7-9}$ in the study done by Khursheed APGAR score at birth was low $(<6)$ in $44(30.98 \%)$ babies of reactive group and 33 $(53.22 \%)$ babies of non-reactive group. ${ }^{9}$ The results were statistically significant, meaning that CTG can detect poor APGAR in compromised fetuses.

In our study $12(12 \%)$ babies among abnormal group and $2(2 \%)$ among normal group needed resuscitation in the form of bag and mask ventilation and oxygen via facemask with a statistically significant $\mathrm{p}$ value of 0.005 . Common causes for need of neonatal resuscitation were meconium aspiration syndrome, birth asphyxia or perinatal depression. Khursheed had $30.98 \%$ cases in the normal group and $53.33 \%$ cases in the abnormal group that needed resuscitation. ${ }^{9}$ Similar results were seen in studies by Banu et al where $15.6 \%$ and Tan et al where $12.8 \%$ babies in the abnormal group required resuscitation.

$12(12 \%)$ babies among abnormal group and $1(1 \%)$ among normal group got admitted in neonatal ICU. Reason for admission in the normal group was for observation in view of respiratory distress and grunting, while that in the abnormal group was meconium aspiration, perinatal depression, birth asphyxia.

Behuria had $29 \%$ NICU admission in the normal group and $64 \%$ in the abnormal group, while Khursheed showed $16.9 \%$ admission in the normal group and $30.64 \%$ in the abnormal group.

One major reason for the NICU admissions to be so less compared to the other studies was that we refer a lot of cases to other centres due to unavailability of bed in our centre, and these cases have not been counted in the study. Moreover, high risk cases had been excluded from the time of initiation of the study, which have been taken into account in the other studies mentioned above.

In the current study the mode of delivery in the abnormal CTG group 90 (90\%) cases had caesarean section, 9 (9\%) had normal vaginal delivery and only 1 (1\%) had instrumental delivery whereas 90 (90\%) had normal vaginal delivery, 7 (7\%) had caesarean section and only 3 (3\%) had instrumental delivery in normal CTG group

The indication of operative delivery being, prolong second stage of labor along with poor maternal effort or non-progress of labor or arrest of descent and dilatation in the normal group, while non-reassuring CTG was the indication of LSCS in the abnormal group.

Paes, in their study of 100 admission tests found that when the admission test was reactive, only one patient developed fetal distress and was delivered by caesarean section with good perinatal outcome. ${ }^{10}$ Five patients delivered by instrumentation for indications other than fetal distress, did so after about 3 hours of the admission test. When the admission test was ominous (9 cases), 8 developed fetal distress out of which 6 had to be delivered by caesarean section and 2 by instrumentation with good perinatal outcome. This study suggests that the admission test might be a good predictor of fetal wellbeing during the next few hours in term fetuses except for acute events which is similar to our study

In Das $\mathrm{V}$ study, $45.7 \%$ cesarean section was done in reactive admission test group and out of this $24 \%$ patients had LSCS for fetal distress. While in patients with abnormal admission test, $60 \%$ had LSCS, of which $47.8 \%$ had LSCS for fetal distress during labor. ${ }^{11}$ This study included both high and low risk patients hence the rate of LSCS is high in both the groups while our study had excluded the high-risk cases.

Chua et al, reported that operative delivery ( $\mathrm{P}<0.001)$, 5min Apgar score $<7$ ( $\mathrm{P}<0.005)$, assisted ventilation ( $\mathrm{P}$ $<0.001)$ and admission to NICU (P <0.001) were significantly associated with abnormal CTG at admission which is comparable with the results of our study. ${ }^{12}$

The study indicates that as the CTG progress from normal to pathological trace the obstetric intervention (instrumental delivery and LSCS) increases. Increased incidence of LSCS in pathological admission test group is because early intervention is taken considering that distressed fetus will not withstand the stress of labor.

Meconium stained liquor was more common in the cases which had non-reassuring pattern. The fetal heart rate pattern is greatly affected by meconium-stained liquor and is poor prognostic factor for perinatal outcome but in our study $40 \%$ cases had meconium stained liquor even though the APGAR score was more than 7 in almost $98 \%$ of all cases, thereby concluding that meconium stained liquor does not necessarily predict fetal distress but can be normal physiological function of the fetus.

In the study of Chua, Meconium Stained Liquor was found in $16 \%$ cases while in the study by Mahomed, meconium staining was $40 \%$ in electronically monitored group, and in our study also $40 \%$ patients had meconium stained amniotic fluid. ${ }^{12,13}$

Among the 200 patients $17(17 \%)$ women in the abnormal CTG group and $10(10 \%)$ women in the normal CTG group had single or multiple loops of umbilical cord wound around fetal neck causing distress to the fetus. 
The study by Begum S (sensitivity 87\%) where the authors have also assessed high risk cases, and that by Xavier AA (sensitivity 90.5\%) have high sensitivity and high negative predictive value that supports the above statement. ${ }^{14,15}$

The high sensitivity of the admission test means that a normal test accurately excludes the presence of adverse fetal status at the time of testing, unless some acute insult to the fetus occurs, which is consistent with the results obtained in our study.

Though most of the above workers recommend cardiotocography as an excellent screening test for detecting fetal distress, some authors doubt its usefulness.

Detractors of electronic fetal monitoring like lmpey believe that neonatal outcome is not significantly improved by the use of admission testing as compared to intermittent fetal heart rate auscultation during labor. ${ }^{16}$ Thacker also feel that the use of electronic fetal monitoring is of limited effectiveness and carries an increased risk of interventions. ${ }^{17}$ According to them increased information at admission will not necessarily lead to better clinical outcomes. This may be true in industrialized countries provided that patients receive comprehensive antenatal care and personal attention during labor. The same does not hold true for our set up where the antenatal care is inadequate and deliveries are conducted in crowded settings and inadequate health care provider to patient ratios.

Desai and Gandhvi proved in their study that CTG has poor sensitivity in screening for asphyxia in fetus with a high negative predictive value while in our study the sensitivity was high $(74 \%) .{ }^{18}$

Gourounti K, Sandall J, concluded that intrapartum admission cardiotocography in women at low obstetric risk increases the risk of caesarean section and instrumental delivery. ${ }^{19}$ In addition, there is no evidence for neonatal benefit in terms of APGAR score at 5 min after delivery. While in our study CTG predicted APGAR score at 5 minutes after delivery in patients in the abnormal CTG group less than 5 in $1 \%$, between $5-7$ in $3 \%$ and more than 7 in $96 \%$ patients respectively, which shows that all the fetuses shown to be compromised on cardiotocography may not be compromised and can have a good prognosis.

\section{CONCLUSION}

CTG is a simple, cost effective and non-invasive test in admission that can serve as a screening tool in triaging fetuses in distress or those who are likely to develop distress, thereby preventing unnecessary delay in intervention. This study tries to study the ability of the test to identify or predict the fetal distress at the time of admission.
Cardiotocography as admission test showed sensitivity of $85.7 \%$ and specificity of $52.7 \%$ to predict the need for resuscitation, sensitivity of $92.3 \%$ and specificity of $52.9 \%$ to predict the need for neonatal admission, sensitivity of $90.0 \%$ and specificity of $91.0 \%$ to predict the mode of delivery and sensitivity of $75.0 \%$ and specificity of $66.6 \%$ to predict the presence of meconium stained liquor in the present study which is highly significant statistically.

There is a significant relationship between CTG results and fetal distress as measured by APGAR scores, need for resuscitation, neonatal admission and operative intervention.

From an analysis of this study, it would be safe to conclude that an ominous admission test should be managed appropriately without delay and obstetrician should be vigilant in suspicious as well as in normal admission test group by doing continuous or intermittent monitoring for timely intervention.

\section{ACKNOWLEDGMENTS}

Authors would like to thank all the doctors, nurses, staffs, of Department of Obstetrics and Gynaecology for their kind support during the study. Authors would like to express our sincere gratitude and respect to all patients who participated in the study.

Funding: No funding sources Conflict of interest: None declared

Ethical approval: The study was approved by the Institutional Ethics Committee

\section{REFERENCES}

1. Haws RA, Yakoob MY, Soomro T, Menezes EV, Darmstadt GL, Bhutta ZA. Reducing stillbirths: screening and monitoring during pregnancy and labour. BMC pregnancy and childbirth. 2009;1:S5.

2. Sandhu GS, Raju R, Bhattacharyya TK. Admission cardiotocography screening of high-risk obstetric patients. Med J Arm Forc India. 2008;64(1):43-5.

3. Gupta M, Nagar T, Gupta P. Role of cardiotocography to improve perinatal outcome in high risk pregnancy. IJCMR. 2017;4(4):853-6.

4. Arulkumaran SS, Rohan D' Souza. Intrapartum fetal monitoring In: The management of labour, 3rd edition, Hyderabad: Universities Press (India); 2011:85-111.

5. Parer JT, King T. Fetal heart rate monitoring: is it salvageable? Am J Obstet Gynaecol. 2000;182(4):982-7.

6. Prabha S, Jha K. Role of admission cardiotocography in predicting perinatal outcome in low risk obstetric population. IJMRP. 2017;3(2):3-6.

7. Behuria S, Nayak R. Admission cardiotocography as a screening test in high risk pregnancies and its co- 
relation with peri-natal outcome. Int J Reprod Contracept Obstet Gynecol. 2016;5(10):3525-8.

8. Goyal SG, Shah PT, Deliwala KJ. Role of fetal monitoring in high risk pregnancy by fetal electrocardiogram, Int $\mathbf{J}$ Reprod Contracept Obstet Gynecol. 2014;3(4):893-7.

9. Khursheed F, Chandra MD, Jatoi N. Cardiotocography: obstetric and neonatal outcome. J Rawalpindi Med College. 2009;13(2):86-8.

10. Paes DF, Mataliya MA, Mhatre PN. The admission test and its relation with labour outcome. Obstet Gynaecol Ind. 1996;46:324-7.

11. Vinita D, Katiyar N, Malik GK. Role of admission test. J Obstet Gynae. 2002;51(1):48-50.

12. Chua S, Arulkumaran S, Kurup A, Anandakumar C, Selemat N, Ratnam SS. Search for the most predictive tests of fetal well-being in early labor. J Peri Med-Official J WAPM. 1996;24(3):199-206.

13. Mahomed J. Intrapartum fetal heart rate monitoringcontinuous electronic versus intermittent doppler-a randomised controlled trial. Central African J Med. 1992;38(12):458-62.

14. Sultana J, Chowdhury TA, Begum K, Khan MH. Comparison of normal and abnormal cardiotocography with pregnancy outcomes and early neonatal outcomes. Mymensingh medical journal: MMJ. 2009;18(1 Suppl):S103-107.
15. Xavier AA, Pandey D, Dogra L, Lewis LE. Cardiotocography in a perinatal armamentarium: boon or bane?. Int J Reprod Contracept Obstet Gynecol. 2017;4(6):2000-4.

16. Impey L, Reynolds M, MacQuillan K, Gates S, Murphy J, Sheil O. Admission cardiotocography: a randomised controlled trial. The Lancet. 2003;361(9356):465-70.

17. Thacker SB, Stroup D, Chang MH, Henderson SL. Continuous electronic heart rate monitoring for fetal assessment during labor. Cochrane Database Systematic Reviews. 2001:2.

18. Desai P, Gandhvi U. Admission CTG as a screening test for fetal distress: not reliable. J Obstet Gynecol Ind. 2004;54:3:260-2.

19. Gourounti, K, Sandall J. Admission cardiotocography versus intermittent auscultation of fetal heart rate: Effects on neonatal APGAR score, on the rate of caesarean sections and on the rate of instrumental delivery: a systematic review. Int J Nurs Stud. 2007;44(6):1029-35.

Cite this article as: Faruqi M, Shrestha SC, Bhatta RD. Pregnancy outcomes of normal versus abnormal cardiotocography in a tertiary centre in Nepal. Int $\mathbf{J}$ Reprod Contracept Obstet Gynecol 2019;8:4406-13. 